\title{
Non-SMC condensin I complex subunit H (NCAPH), a regulator of cell cycle, predicts poor prognosis in lung adenocarcinoma patients: a study mainly based on TCGA and GEO database
}

\author{
Wei Zhou ${ }^{1,2}$, Jia Hu${ }^{3,4}$, Jun Zhao ${ }^{1}$ \\ ${ }^{1}$ Department of Thoracic Surgery, The First Affiliated Hospital of Soochow University, Suzhou, China; ${ }^{2}$ Department of Cardiothoracic Surgery, The \\ First Affiliated Hospital of Wannan Medical College, Wuhu, China; ${ }^{3}$ Department of Neurology, The First Affiliated Hospital of Wannan Medical \\ College, Wuhu, China; ${ }^{4}$ Department of Neurology, First Affiliated Hospital of Soochow University, Suzhou, China \\ Contributions: (I) Conception and design: W Zhou, J Zhao; (II) Administrative support: J Hu; (III) Provision of study materials or patients: W Zhou; \\ (IV) Collection and assembly of data: W Zhou; (V) Data analysis and interpretation: W Zhou; (VI) Manuscript writing: All authors; (VII) Final \\ approval of manuscript: All authors. \\ Correspondence to: Jun Zhao. Department of Thoracic Surgery, First Affiliated Hospital of Soochow University, 899 Pinghai Road, Gusu District, \\ Suzhou 215006, China. Email: zhaojunsuda@163.com.
}

Background: Lung adenocarcinoma (LUAD) is the main sub-type of lung cancer, which is a major disease
of human death. However, the role of non-SMC condensin I complex subunit H (NCAPH) in LUAD and its
possible upstream regulation microRNAs (miRNAs) remains unclearly.
Methods: In this study, we analyzed the NCAPH mRNA and protein expression in normal and cancer
tissues mainly based on Human Protein Atlas (HPA) database, The Cancer Genome Atlas (TCGA) and
Gene Expression Omnibus (GEO) databases. With the help of the Kaplan Meier plotter, we explored the
prognosis role in LUAD. Furtherly, the co-expressed genes of NCAPH in LUAD were obtained by using
cBioPortal, GEPIA and UALCAN database. Then, gene ontology (GO) and Kyoto Encyclopedia of Genes
and Genomes (KEGG) pathway analysis of co-expression genes of NCAPH was conducted by DAVID,
while the protein-protein interaction (PPI) network was constructed with STRING and hub genes were
identified and visualized by Cytoscape software. We also investigated the miRNAs and chemicals that may
downregulated the NCAPH expression. Results: The results showed that NCAPH expression level was elevated in LUAD tissue compared with normal lung tissue and predicted poor prognosis. GO and KEGG pathway enriched analysis of co-expressed genes suggested that NCAPH may play an important role in cell cycle in LUAD. Nine top hub co-expressed genes were all negatively related to the LUAD prognosis. Lastly, 8 miRNAs and 5 chemicals were identified to have the potential to down-regulate the NCAPH expression.

Conclusions: Our study indicated that NCAPH expression in LUAD is a poor prognostic indicator, which may be the potential therapeutic target in the future.

Keywords: lung adenocarcinoma (LUAD); non-SMC condensin I complex subunit H (NCAPH); miRNA; prognosis

Submitted May 29, 2020. Accepted for publication Oct 21, 2020.

doi: $10.21037 /$ tcr-20-2217

View this article at: http://dx.doi.org/10.21037/tcr-20-2217

\section{Introduction}

Lung cancer, the leading cause of cancer-related mortality, is a major disease that threatens human health (1-4). As far as the progression in surgery, radiotherapy and chemotherapy, the 5 -year survival rate of lung cancer is only $15 \%$ (5). However, the adoption of immunotherapies and targeted 
therapies have increased the 5 -year survival rate obviously (range from $15 \%$ to $50 \%$ ) (6,7). Lung adenocarcinoma (LUAD) is the main sub-type of lung cancer, and in recent years the incidence of LUAD has increased distinctly. So, it's obliged to find new therapeutic targets for LUAD.

Condensin, a multiprotein complex, serves as a regulator of chromosome-wide gene. During mitosis and meiosis, It plays an important role in the process of chromosome assembly and segregation, regulating the cell cycle (8). There are two kinds of condensin complex: condensin I and condensin II. Condensing I complex is composed of structural maintenance of chromosomes (SMC) proteins and three non-SMC subunits, including non-SMC condensin I complex subunit $\mathrm{H}(\mathrm{NCAPH})$, subunit I (NCAPG) and subunit D2 (NCAPD2) (9). NCAPH is upregulated in many kinds of tumor tissue, such as prostate cancer, pancreatic cancer and hepatocellular carcinoma, and it often predicts poor prognosis (10-12), regulating the cell cycle, migration and invasion. These studies proved that NCAPH could be an important therapeutic target for cancer. But in colon cancer, NCAPH is positively correlated with prognosis (13). Till now, the exact role of NCAPH in LUAD and its possible upstream regulation mechanism remain unclearly.

In order to answer this question, The Cancer Genome Atlas (TCGA) database and Gene Expression Omnibus (GEO) database were analyzed with online tools. We demonstrate that NCAPH is up-regulated in LUAD compared with adjacent normal tissues. What's more, the survival analysis revealed that LUAD patients with high level of NCAPH had worse survival time. Potential mechanism and its upstream microRNAs (miRNAs) were also analyzed by online tools, such as cBioPortal, GEPIA, UALCAN and TargetScan. In conclusion, our study offers a new potential therapeutic target for the treatment of LUAD. We present the following article in accordance with the TRIPOD reporting checklist (available at http://dx.doi. org/10.21037/tcr-20-2217).

\section{Methods}

\section{Analysis of TCGA and GEO database}

TCGA database (https://www.cancer.gov/tcga), a landmark cancer genomics program, was created by the National Cancer Institute and the National Human Genome Research Institute in 2006. It includes genomic, epigenomic, transcriptomic, and proteomic data of 33 cancer types, as well as the matched normal samples. In order to view the expression profile of NCAPH in pancancer, we use a web-based database-GEPIA (http://gepia. cancer-pku.cn). This database could analyze the RNA sequencing expression data from the TCGA and the GTEx projects (14). For LUAD patients, the relationship between clinicopathological parameters and NCAPH expression was explored by UALCAN (http://ualcan.path.uab.edu/ index.html) (15), which is an interactive and user-friendly web resource mainly based on TCGA data. The enrolled clinicopathological features included gender, age, tumor stage, lymph node metastasis status and smoking history.

GEO database (https://www.ncbi.nlm.nih.gov/geo/) was furtherly mined for demonstrate the deferent expression level of NCAPH mRNA between the LUAD tissue and normal lung tissue. GEO database is a good helper for providing users with array- and sequence-based data. In this study, the RNA-seq data of GSE19188 (16), GSE7670 (17) and GSE10072 (18) were extracted and analyzed by GEO2R online tool. The study was conducted in accordance with the Declaration of Helsinki (as revised in 2013).

\section{Human Protein Atlas (HPA) database analysis}

Expression level of NCAPH mRNA and protein in human normal organs were retrieved by HPA database (https:// www.proteinatlas.org) (19). This database contains immunohistochemistry (IHC) staining pictures of 20 LUAD tissues and 6 normal lung tissues. According to the IHC staining intensity, the picture was scored from 0 to 3 (0, negative; 1 , weak staining; 2 , moderate staining; 3 , strong staining). Based on the percentage of stained cells, the picture was scored from 0 to 3 ( 0 , none; $1,<25 \% ; 2$, $25-75 \% ; 3,>75 \%)$. The IHC score for every picture was calculated by multiplying the staining intensity score with the staining extent score, so the IHC score ranges from 0 to 12 for each sample.

\section{The Kaplan Meier plotter database analysis}

The Kaplan Meier plotter (https://kmplot.com/analysis/) includes gene chip and RNA-seq data of 20 different cancer types. The system data mainly comes from GEO and TCGA database, which can make a meta-analysis based discovery and validation of survival biomarkers (20). By using the Kaplan Meier plotter database, we examined the relationship between NCAPH mRNA expression and the overall survival 
(OS) time of 720 LUAD patients. All the patients were divided into two groups (high $v s$. low) by median expression value of NCAPH mRNA. Then the web-based tool can calculate the log-rank $\mathrm{P}$ value, hazard ratio (HR), and $95 \%$ confidence interval (CI) directly. OS time, FP (first progression time), and PPS (post progression survival time) were used to estimate the prognosis of LUAD.

\section{GO and KEGG pathway analysis of co-expression genes of $\mathrm{NCAPH}$}

Firstly, we explored the co-expressed genes of NCAPH in LUAD patients using cBioPortal database (http://www. cbioportal.org/). With the help of cBioPortal database, we can download the co-expressed genes of NCAPH in LUAD patients based on TCGA data (21). In order to obtain the accurate co-expressed genes, we used two other databases: GEPIA and UALCAN. Then we intersected the results obtained from these three databases through a website toolVENNY (https://bioinfogp.cnb.csic.es/tools/venny/).

Secondly, for the aim to understand the potential biological process and pathway, the terminal results of co-expressed genes were furtherly processed with gene ontology (GO) and Kyoto Encyclopedia of Genes and Genomes (KEGG) pathway analysis. The Database for Annotation, Visualization and Integrated Discovery (DAVID) (https://david.ncifcrf.gov) was accepted for these analyses (22). We visualized the GO and KEGG analysis results using $\mathrm{R} /$ Bio conductor (version 3.26.5).

\section{PPI network construction and bub-gene analysis}

STRING (version 11.0) is a web-based tool (https://stringdb.org) for exploring the known and predicted proteinprotein interactions (PPI) (23). We inputted the coexpressed genes of NCAPH in LUAD patients into the STRING database, then it could analyze the PPI network automatically. In order to get the hub-genes in the PPI network, the cytoHubba app of Cytoscape software (version 3.6.1) was utilized. The top nine genes ranked by degree were calculated and visualized (24).

\section{Prediction of potential miRNAs that regulate NCAPH}

TargetScan (version 7.2, http://www.targetscan.org/ vert_72/) predicts potential miRNAs for mRNA by searching for the seed region of miRNA that matches to human 3' UTRs (25). OncomiR database (http://www. oncomir.org/) is an open-source platform for pan-cancer miRNA dysregulation in cancer (26). MiRNAs and its target genes are negatively correlated. So, we searched OncomiR database for down-regulated miRNAs in LUAD tissues. To further narrow down the range of target genes, miRNAs positively related with survival time were also analyzed using OncomiR database. Then, we got 9 common miRNAs of the three data sets. ENCORI (http://starbase. sysu.edu.cn/) was an online tool for analyzing TCGA RNASeq data of many kinds of cancers including LUAD (27). Then, we used ENCORI database to assess the expression correlation of NCAPH mRNA and these 9 miRNAs.

\section{The analysis of potential chemicals that down-regulate $\mathrm{NCAPH}$}

CTD (http://ctdbase.org/) is a publicly available database that can be used to analyze the chemical-gene interactions (28). We searched chemicals that could down-regulate NCAPH mRNA or protein. Chemicals with at least 2 references were treated as potential drugs. The structure of potential chemicals was furtherly downloaded by using PubChem database (https://pubchem.ncbi.nlm.nih.gov/).

\section{Statistical analysis}

Statistics analysis were carried out using GraphPad Prism 7, and the results are presented as the mean $\pm \mathrm{SD}$. Differences between two groups was evaluated by Student's $t$-test (unpaired, two-tailed). One-way ANOVA was used for the data subject to normal distribution. $\mathrm{P}<0.05$ was considered significant.

\section{Results}

\section{Expression levels of NCAPH in buman normal and cancer tissues}

In order to clarify the expression level of NCAPH mRNA in normal tissues, the HPA database was used. The results proved that the level of NCAPH mRNA was very low in many normal tissues, such as lung, cerebral cortex, adipose tissue, cervix, and uterine, etc. (Figure 1A). However, in LUAD as well as many other types of cancers, the NCAPH mRNA expression level increased significantly in comparison with normal tissues (Figure 1B). 
A
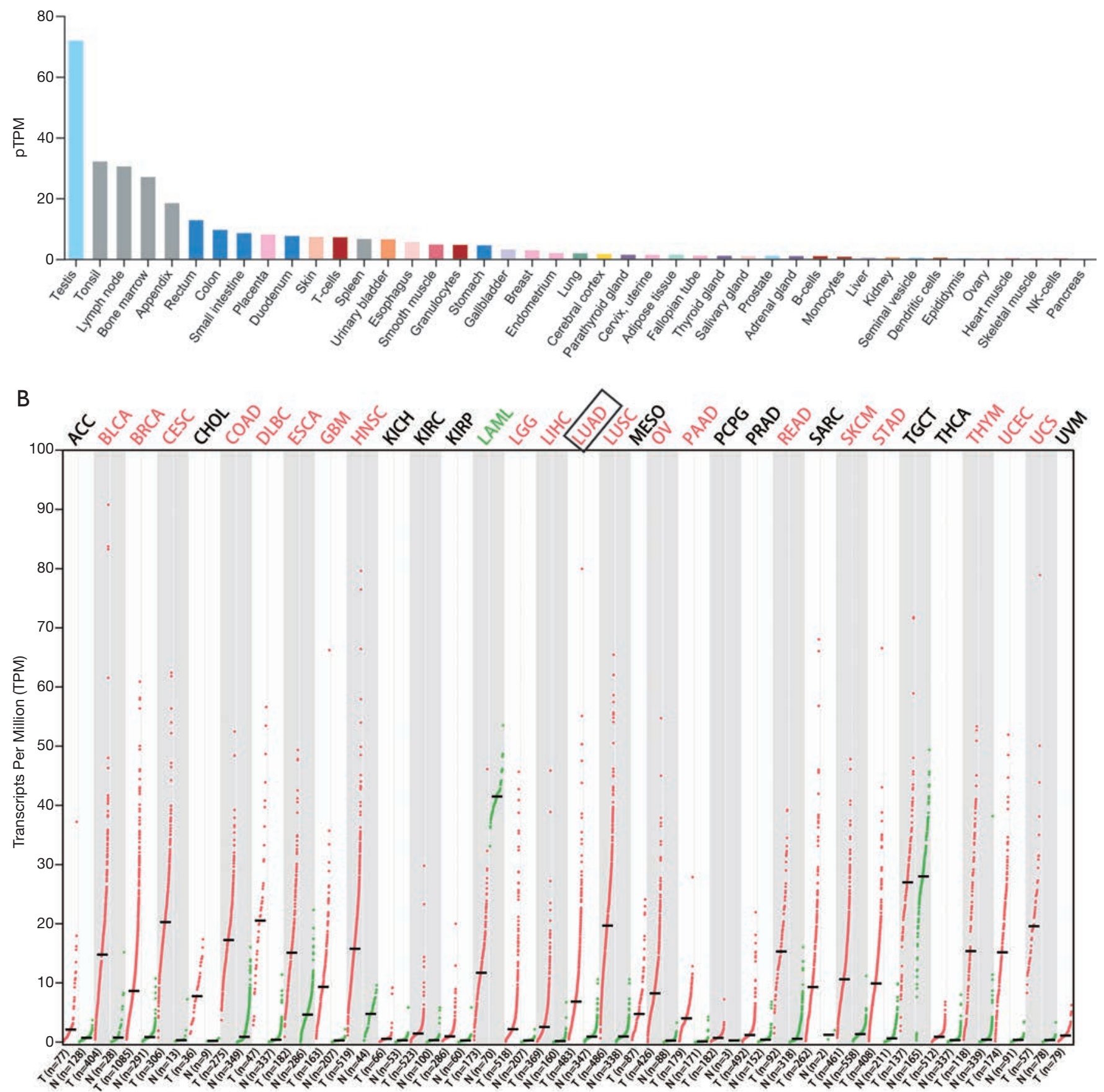

Figure 1 Expression level of NCAPH mRNA in normal and cancer tissues. (A) Expression profile of NCAPH mRNA in normal tissues based on Human Protein Atlas (HPA) database. (B) Expression profile of NCAPH mRNA in cancer tissues based on GEPIA database. Black box pointed NCAPH mRNA expression level in lung adenocarcinoma (LUAD). 


\section{Elevated NCAPH mRNA in LUAD based on TCGA and $G E O$}

ULCAN, the online analysis tool of TCGA, showed that NCAPH mRNA expression level was higher in LUAD (Figure 2A). Then we investigated the relationship between the NCAPH mRNA in LUAD and the clinicopathological parameters. LUAD patients from stage I to stage IV got a higher level of NCAPH mRNA compared with normal lung tissues (Figure 2B). The results showed that patients with all kinds of subtype such as nodal metastasis status (Figure 2C), gender (Figure 2D), age (Figure 2E), and smoking habits (Figure $2 F$ ) got higher NCAPH mRNA levels compared with normal lung tissues. We also explored the expression of NCAPH mRNA in GEO database. GSE19188, GSE7670 and GSE10072 were enrolled in our study. NCAPH mRNA levels were relatively higher in LUAD patients in the three datasets (Figure 2G,H,I).

\section{Increased expression of NCAPH protein in LUAD}

Proteins of cells are the fundamental of biological function. In order to study the expression of NCAPH protein, we check the HPA database. There are two kinds of antibody for NCAPH: HPA002647 and HPA003008. Each antibody staining group concluded 3 IHC images of normal lung tissue and 10 IHC images of LUAD tissue. As shown in Figure 3, the IHC images of LUAD got a higher IHC score compared with normal tissues. This result demonstrated that the expression level of NCAPH protein was increased in LUAD tissues in comparison with normal lung tissues.

\section{The prognostic role of NCAPH in LUAD patients}

The prognostic role of NCAPH In LUAD patients was mined by using the Kaplan Meier plotter database. The probe name of NCAPH was 212949_at. As shown in Figure 4, increased level of NCAPH indicated a significantly worse prognosis in the aspects of OS time (Figure $4 A$ ) and FP time (Figure 4B). But, no significant difference was seen between low and high NCAPH expression patients in PPS time (Figure $4 C$ ).

\section{GO functional, KEGG patbway enrichment and PPI network analysis of co-expressed genes of $\mathrm{NCAPH}$}

As shown in Figure $5 A$, we analyzed the co-expressed genes of NCAPH using three databases: UALCAN, cBioPortal and GEPIA. The number of co-expressed genes in the three databases were 3,182, 13,480 and 200 separately. One hundred and seventy-five co-expressed genes were in commonly shared. In order to understand the function of these genes, GO functional analysis (cellular component, molecular function and biological process) and KEGG pathway enrichment analysis were performed by using DAVID database (Table 1).

The top ten GO terms were enriched shown in Figure $5 B$ (cellular component), Figure $5 C$ (molecular function) and Figure 5D (biological process). The mainly enriched items for cellular component were chromosome, chromosomal part, intracellular non-membrane-bounded organelle, non-membrane-bounded organelle and spindle. As far as molecular function items, ATP binding, adenyl nucleotide binding, purine nucleoside binding and nucleoside binding were significantly enriched. The co-expressed genes mainly participated the biological processes of cell cycle, cell cycle phase, $M$ phase, cell cycle process, and mitotic cell cycle. The mainly enriched KEGG pathway items were cell cycle, DNA replication, oocyte meiosis, progesterone-mediated oocyte maturation, and mismatch repair (Figure 5E).

The PPI network analysis was performed by using STRING database and Cytoscape software, containing 175 nodes and 7,840 edges. To find out the key role genes in the network, the cytoHubba app based on Cytoscape software was utilized. Nodes' degree were calculated and ranked by degree from high to low. The top 9 genes were $C D K 1$, BUB1, BUB1B, CCNB1, CCNA2, KIF11, TOP2A, CDC45, $C D C 20$ (Figure 5F).

\section{Validation of the prognosis role of co-expression bub genes}

We searched the GEPIA database to furtherly validate the prognosis role of co-expression hub genes. As shown in Figure 6, The top 9 genes, CDK1, BUB1, BUB1B, CCNB1, CCNA2, KIF11, TOP2A, CDC45, and CDC20, acted as onco-gene role in LUAD. Patients with high level of these gene were proved to have worse prognosis compared with that with low level.

\section{Prediction of miRNAs that regulate NCAPH}

To predict miRNAs that regulate NCAPH, TargetScan database was used to find the potential miRNAs. The results contained 1,075 miRNAs with conserved sites or poorly conserved sites. After searching OncomiR database, we found 123 miRNAs with significantly increased expression 

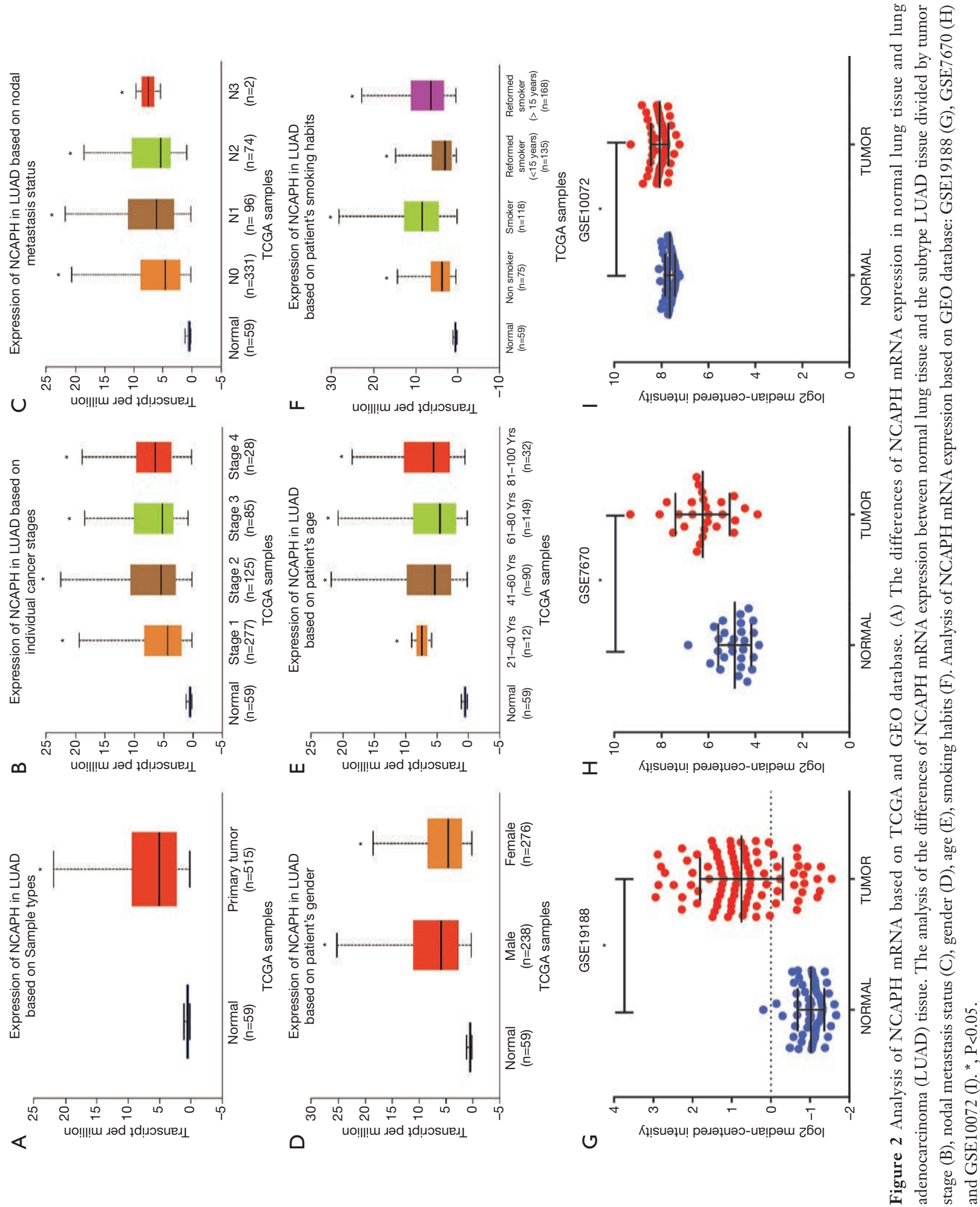

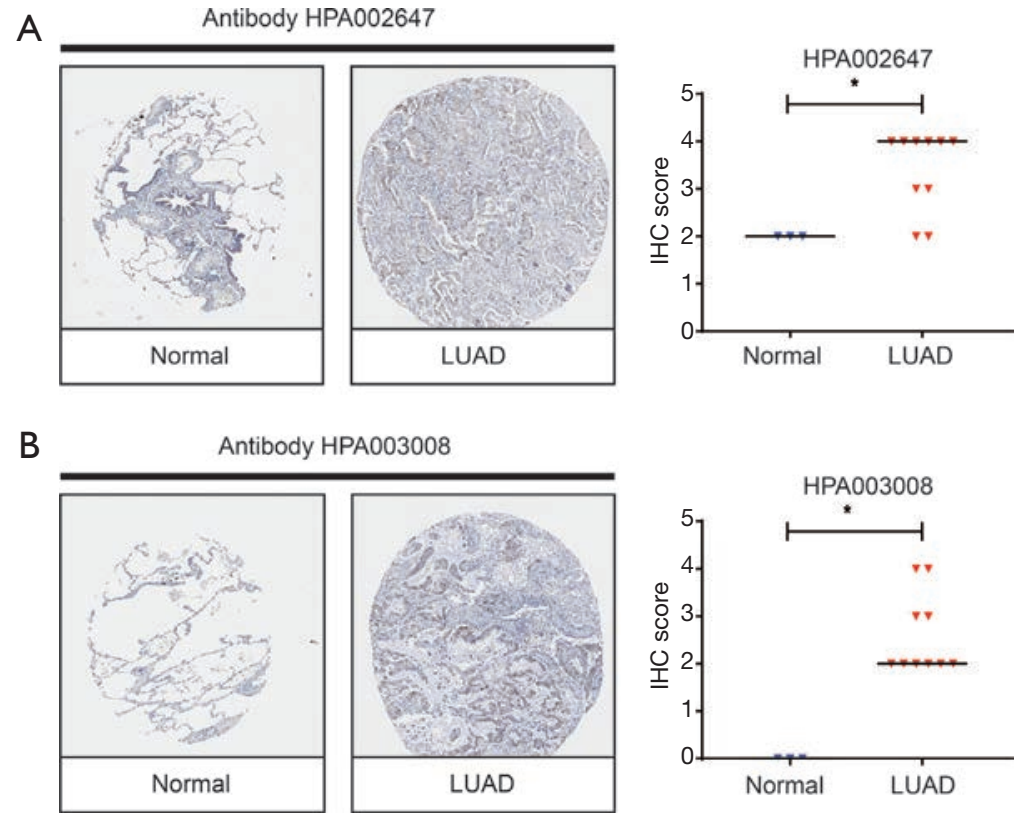

Figure $3 \mathrm{NCAPH}$ protein expression level in normal tissues and Lung adenocarcinoma (LUAD) tissues. (A,B) Based on Human Protein Atlas (HPA) database, the immunohistochemistry (IHC) images were download and then calculated IHC score. (A) antibody HPA002647. (B) antibody HPA003008. *, $\mathrm{P}<0.05$. The blue and red triangles represent IHC scores of normal lung tissue and LUAD, respectively. The magnification of the cell images was $100 \times$.

level in LUAD. Moreover, there were 66 miRNAs that had negative correlated relationship with the prognosis in LUAD based on Oncomir. After we drawn the Venn picture of the three miRNA lists, 9 miRNA were found in common: miR-1976, miR-1468-5p, miR-195-3p, miR-4903p, miR-133b, miR-497-5p, miR-195-5p, miR-125a-5p, miR-500a-3p (Figure 7A). Then, we explored the expression relationship between NCAPH mRNA and each miRNA of the 9 miRNAs. miR-1976, miR-1468-5p, miR-1953p, miR-490-3p, miR-133b, miR-497-5p, miR-195-5p, and miR-125a-5p had negative correlation with NCAPH mRNA $(\mathrm{r}<0, \mathrm{P}<0.05$, Figure $7 B, C, D, E, F, G, H, I)$ except miR$500 a-3 p(r>0, P<0.05$, Figure $7 f)$. The prognostic values of 8 potential miRNAs in LUAD were shown in Table 2 based on Oncomir database.

\section{Potential chemicals that down-regulate NCAPH}

In order to find out chemicals that suppress the NCAPH, we used the CTD database. As a result, 5 chemicals (cyclosporin A, bisphenol A, methyl methanesulfonate, doxorubicin, valproic acid) were identified to decrease the expression of NCAPH mRNA with $\geq 2$ references. The structure was furtherly downloaded by using PubChem database (Figure 8).

\section{Discussion}

The knowledge about the prognostic role of NCAPH in LUAD is very poor. In this study, we aimed to demonstrate that NCAPH expression level was elevated in LUAD and predicted poor prognosis. Systematic analysis of the NCAPH expression level in LUAD patients was performed by using TCGA, GEO, and HPA databases from two aspects: mRNA level and protein level (29-31). The coexpression genes enriched analysis suggested that $\mathrm{NCAPH}$ may play an important role in cell cycle in LUAD.

Similarly, in prostate cancer, pancreatic cancer and hepatocellular carcinoma, NCAPH is up-regulated and often predicts poor prognosis (10-12). With the help of coexpression network analysis, 9 top enriched co-expressed genes (CDK1, BUB1, BUB1B, CCNB1, CCNA2, KIF11, $T O P 2 A, C D C 45, C D C 20$ ) were distinguished and considered to play important role in cell cycle. Previous studies showed that CDK1 and BUB1 were negatively related with LUAD prognosis (32). CCNB1, CCNA2 and BUB1B were regulators in cell cycle and proliferation (33-35). KIF11 could control bipolar spindle formation and 
A

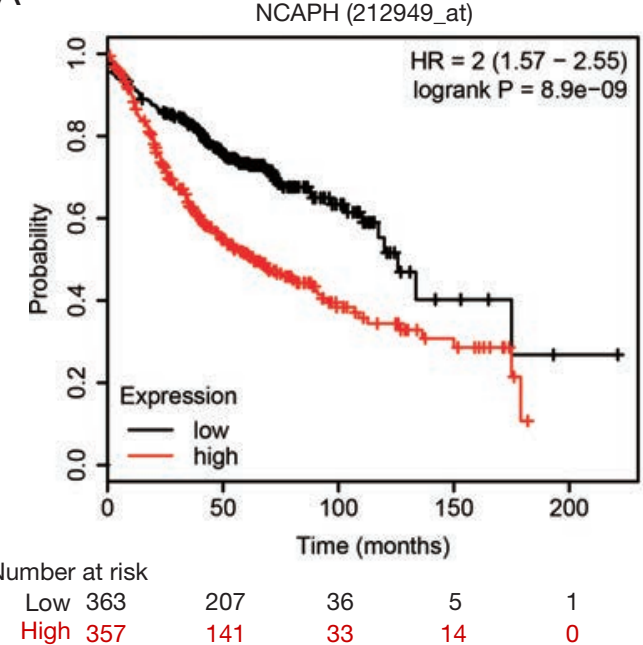

C

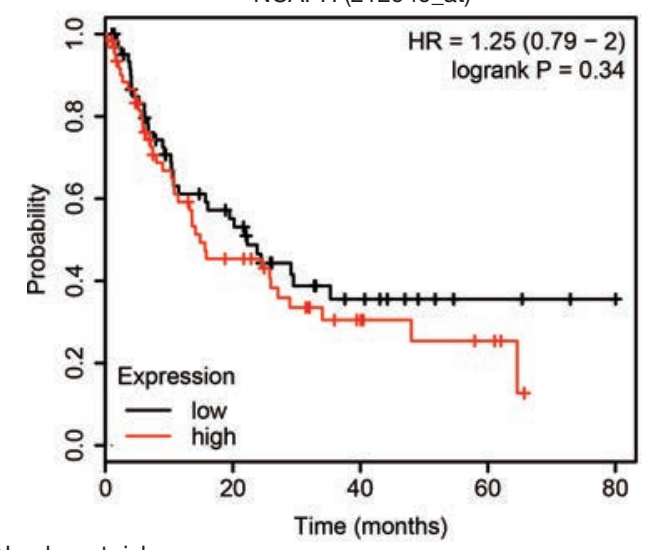

B

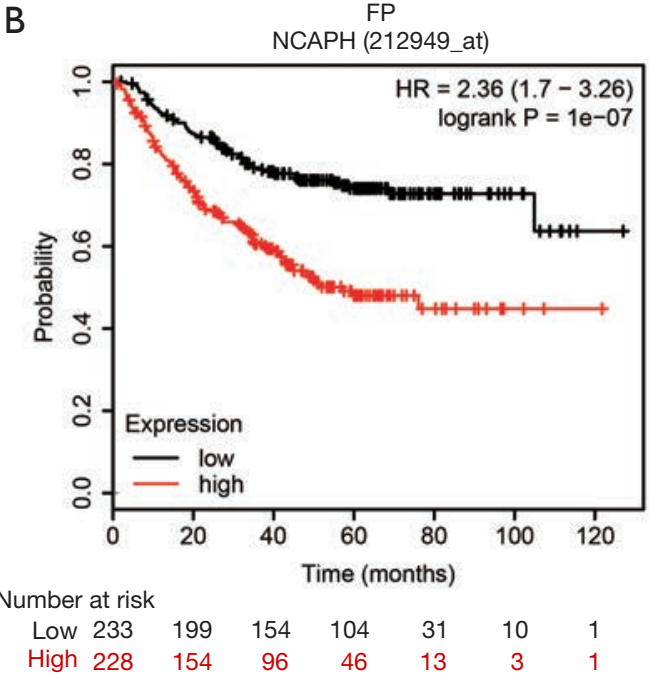

$\begin{array}{ccccc}\text { Number at risk } & & & & \\ \text { Low } 62 & 27 & 10 & 3 & 1 \\ \text { High } 63 & 22 & 8 & 4 & 0\end{array}$

Figure 4 The prognostic analysis of NCAPH. Kaplan-Meier curves of overall survival time (OS) (A), first progression time (FP) (B) and (post progression survival time) PPS (C).

chromosomal stability (36). TOP2A was considered to be a bad prognosis factor in LUAD (37). These previous studies proved the reliability of our conclusions. We argued that NCAPH probably promoted cell proliferation through cell cycle pathway in LUAD. However, elevated expression of NCAPH meant longer OS time in patients with colon cancer (13). The contradiction may be due to the biological differences of variant tumors.

Why was the NCAPH expression level elevated in LUAD. In order to answer this question, we analyzed the miRNAs in LUAD, which could potentially participate in post-transcriptional regulation of many kinds of proteins.
Previous study showed that miRNAs could sponge 3' UTR of its target mRNA (38). We identified 8 aberrantly down-regulated miRNAs that could potentially inhibit the expression of NCAPH. The correlation analysis of miRNAs and NCAPH mRNA was carried out based on ENCORI, which contained TCGA RNA-Seq data of LUAD. So, the results were more convincing. Among these 8 miRNAs, miR-1976, miR-490-3p, miR-133b, miR-497-5p, miR$195-5 p$, miR-125a-5p were previously reported as tumor inhibitors in non-small cell lung cancer (39-44). These results are consistent with our findings. Hence, we assume that NCAPH might be down-regulated by these 8 miRNAs. 
A

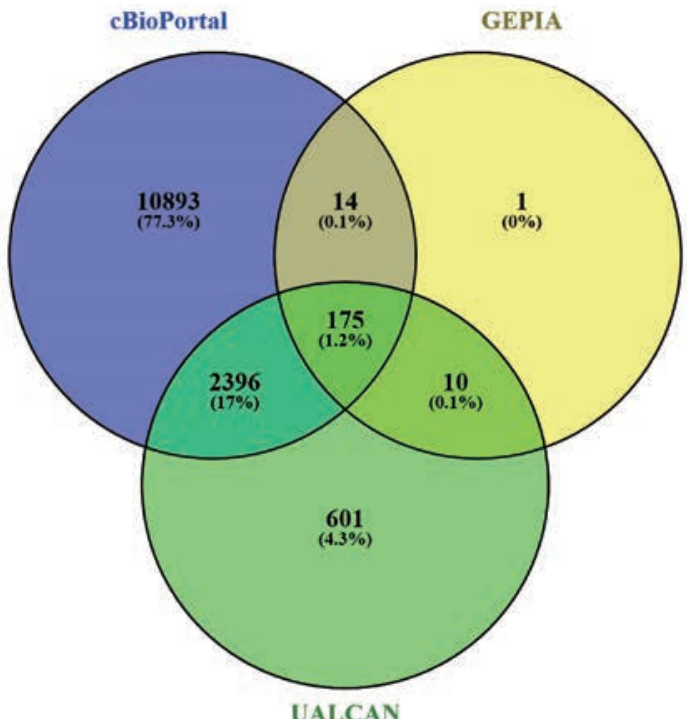

C

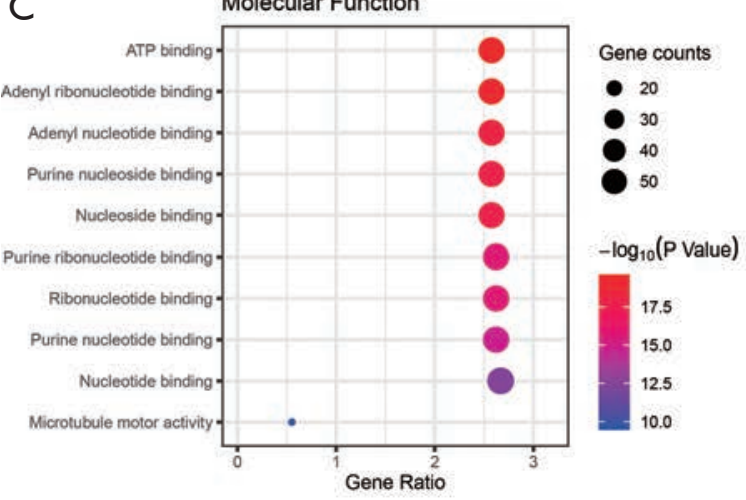

$\mathrm{E}$

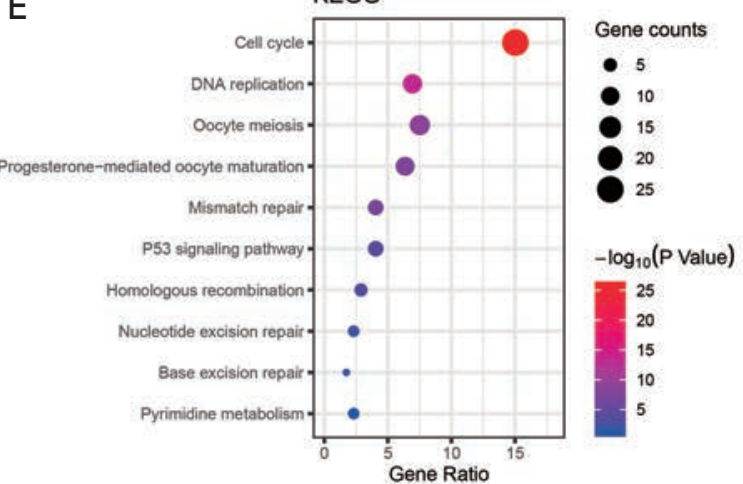

B
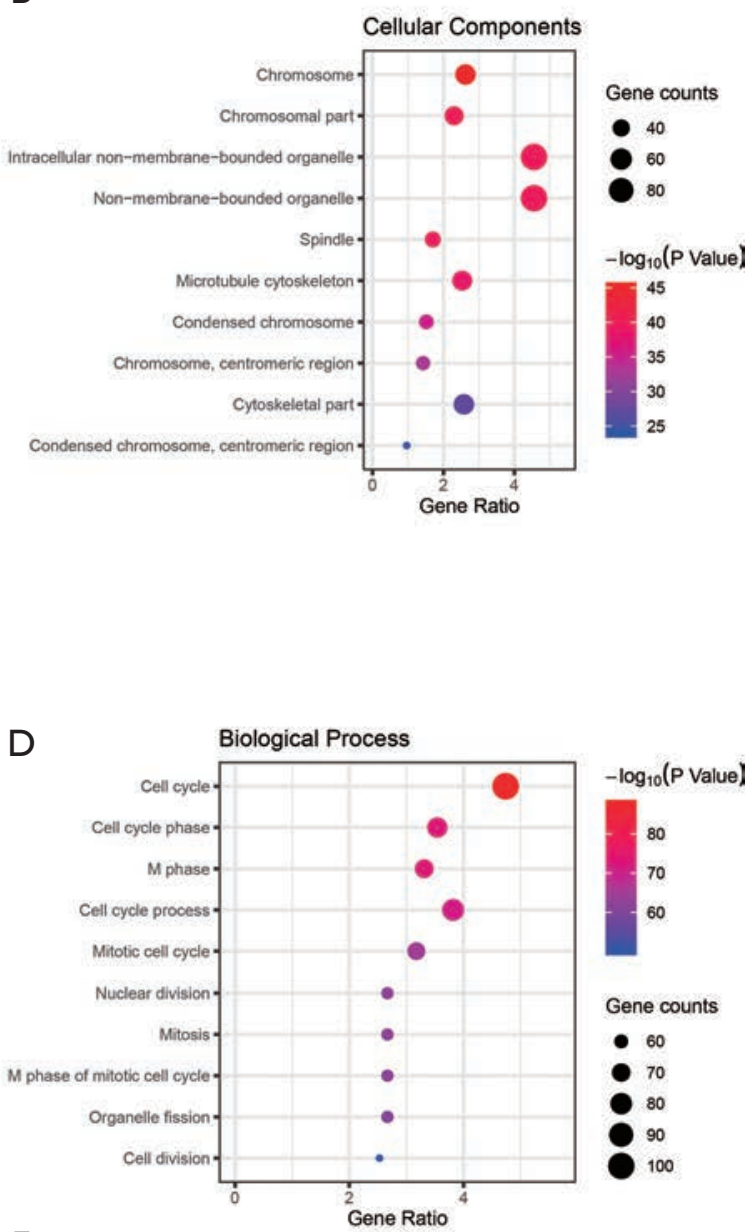

$\mathrm{F}$

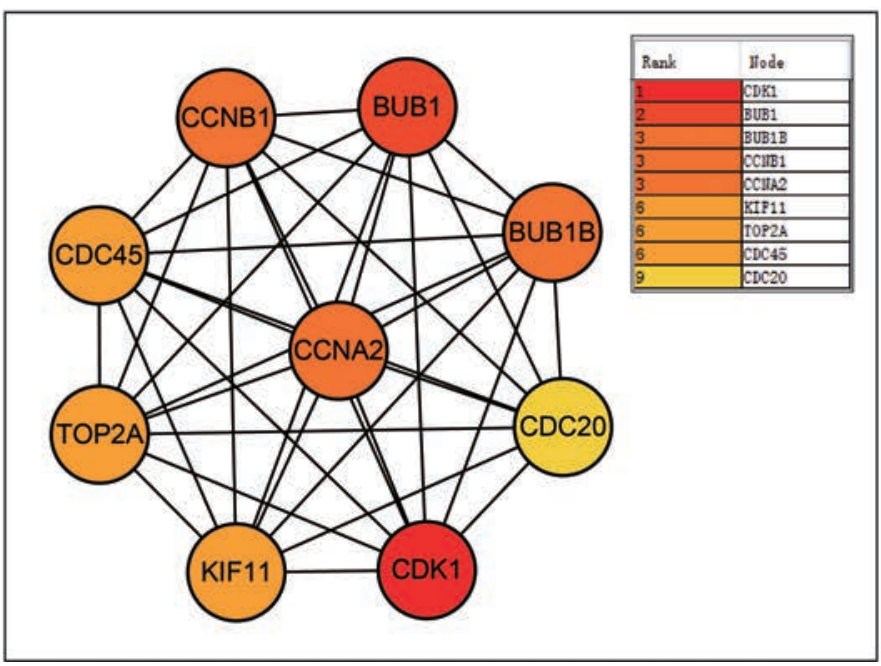

Figure 5 GO functional, KEGG pathway enrichment and PPI network analysis of co-expressed genes of NCAPH. (A) The Venn picture of co-expressed genes of NCAPH based on GEPIA, UALCAN, and cBioPortal database. Go functional analysis of 175 co-expression genes in three functional groups: cellular component (B), molecular function (C) and biological process (D). (E) KEGG pathway enriched analysis of 175 co-expressed genes. (F) Top 9 co-expressed genes based on the calculation of cytoHubba app. 
Table 1 Gene ontology (GO) and Kyoto Encyclopedia of Genes and Genomes (KEGG) analysis of co-expressed genes of NCAPH in lung adenocarcinoma (LUAD)

\begin{tabular}{|c|c|c|c|}
\hline Category & Term & Gene count & $P$ value \\
\hline GOTERM_CC_FAT & GO:0005694-chromosome & 57 & $5.72 \mathrm{E}-46$ \\
\hline GOTERM_CC_FAT & GO:0044427-chromosomal part & 50 & $1.16 \mathrm{E}-40$ \\
\hline GOTERM_CC_FAT & GO:0043228-non-membrane-bounded organelle & 99 & $2.27 \mathrm{E}-40$ \\
\hline GOTERM_CC_FAT & GO:0005819-spindle & 37 & $2.65 \mathrm{E}-40$ \\
\hline GOTERM_CC_FAT & GO:0000793-condensed chromosome & 33 & 5.77E-36 \\
\hline GOTERM_CC_FAT & GO:0000775-chromosome, centromeric region & 31 & 2.39E-33 \\
\hline GOTERM_CC_FAT & GO:0044430-cytoskeletal part & 56 & 7.13E-28 \\
\hline GOTERM_CC_FAT & GO:0000779-condensed chromosome, centromeric region & 21 & $1.35 \mathrm{E}-24$ \\
\hline GOTERM_MF_FAT & GO:0001883-purine nucleoside binding & 56 & $1.75 \mathrm{E}-18$ \\
\hline GOTERM_MF_FAT & GO:0001882-nucleoside binding & 56 & $2.39 \mathrm{E}-18$ \\
\hline GOTERM_MF_FAT & GO:0032555-purine ribonucleotide binding & 57 & 1.69E-16 \\
\hline GOTERM_MF_FAT & GO:0032553-ribonucleotide binding & 57 & $1.69 \mathrm{E}-16$ \\
\hline GOTERM_MF_FAT & GO:0017076-purine nucleotide binding & 57 & $1.53 \mathrm{E}-15$ \\
\hline GOTERM_MF_FAT & GO:0000166-nucleotide binding & 58 & 3.63E-13 \\
\hline GOTERM_MF_FAT & GO:0003777-microtubule motor activity & 12 & $1.96 \mathrm{E}-10$ \\
\hline GOTERM_BP_FAT & GO:0007049-cell cycle & 103 & $1.90 \mathrm{E}-88$ \\
\hline GOTERM_BP_FAT & GO:0000087-M phase of mitotic cell cycle & 58 & 1.23E-62 \\
\hline GOTERM_BP_FAT & GO:0048285-organelle fission & 58 & $5.02 E-62$ \\
\hline GOTERM_BP_FAT & GO:0051301-cell division & 55 & 9.61E-51 \\
\hline KEGG_PATHWAY & hsa04110: cell cycle & 26 & $1.41 \mathrm{E}-26$ \\
\hline KEGG_PATHWAY & hsa03030: DNA replication & 12 & $5.00 \mathrm{E}-14$ \\
\hline KEGG_PATHWAY & hsa04114: oocyte meiosis & 13 & 1.44E-09 \\
\hline KEGG_PATHWAY & hsa04914: progesterone-mediated oocyte maturation & 11 & $2.12 \mathrm{E}-08$ \\
\hline KEGG_PATHWAY & hsa03430: mismatch repair & 7 & 1.19E-07 \\
\hline KEGG_PATHWAY & hsa04115: p53 signaling pathway & 7 & $8.78 \mathrm{E}-05$ \\
\hline KEGG_PATHWAY & hsa03440: homologous recombination & 5 & $2.22 \mathrm{E}-04$ \\
\hline KEGG_PATHWAY & hsa03420: nucleotide excision repair & 4 & 0.012179 \\
\hline KEGG_PATHWAY & hsa03410: base excision repair & 3 & 0.056228 \\
\hline KEGG_PATHWAY & hsa00240: pyrimidine metabolism & 4 & 0.08622 \\
\hline
\end{tabular}


A

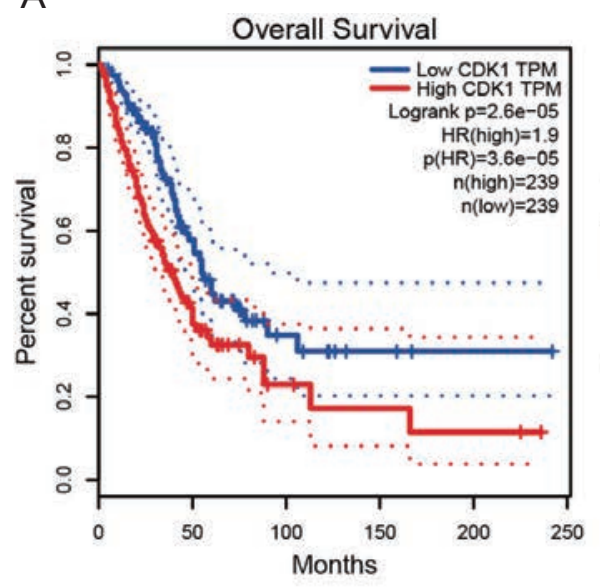

D

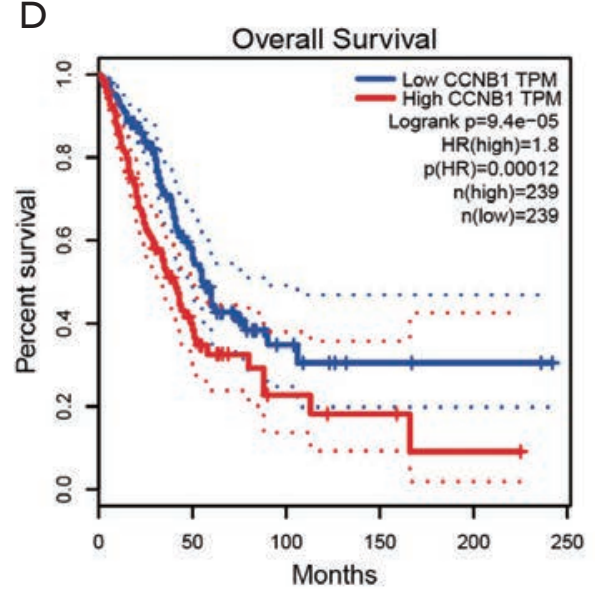

G

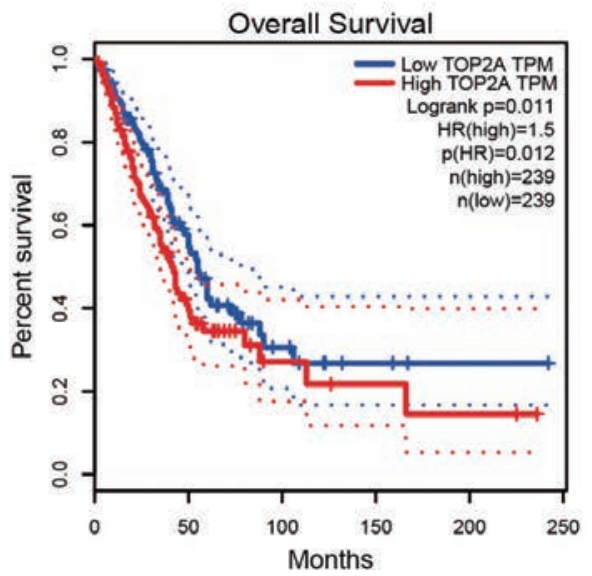

B

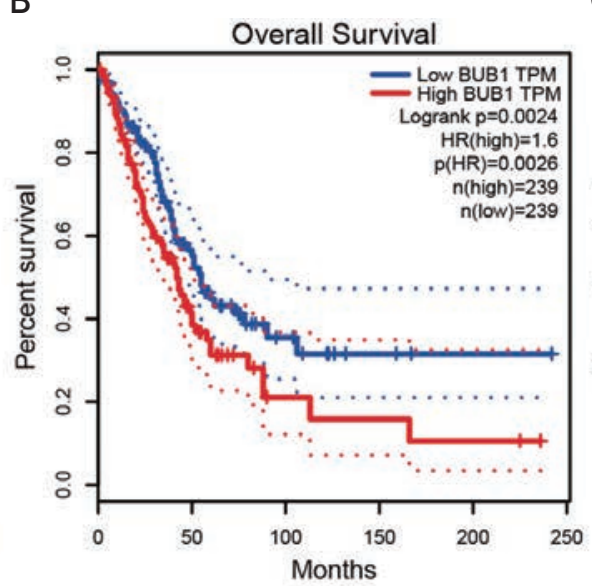

E

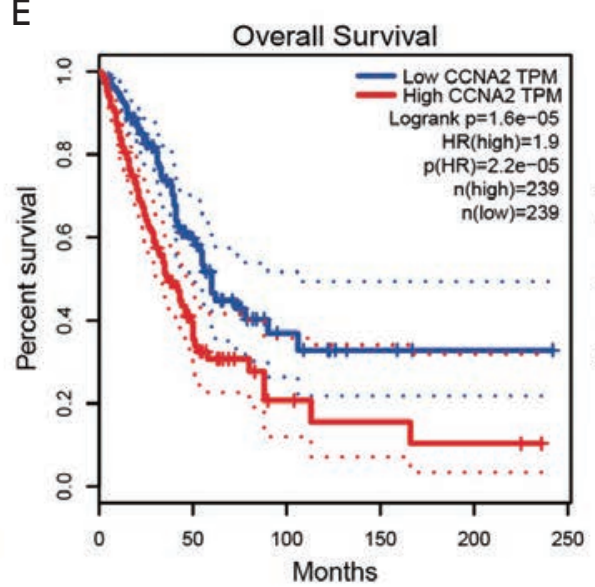

$\mathrm{H}$

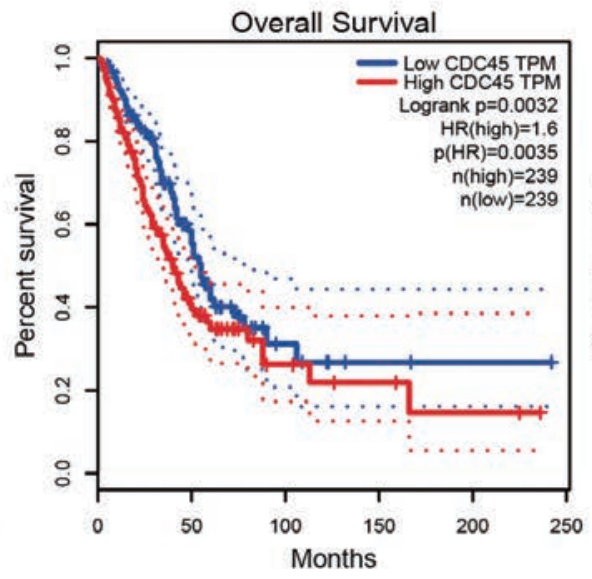

C

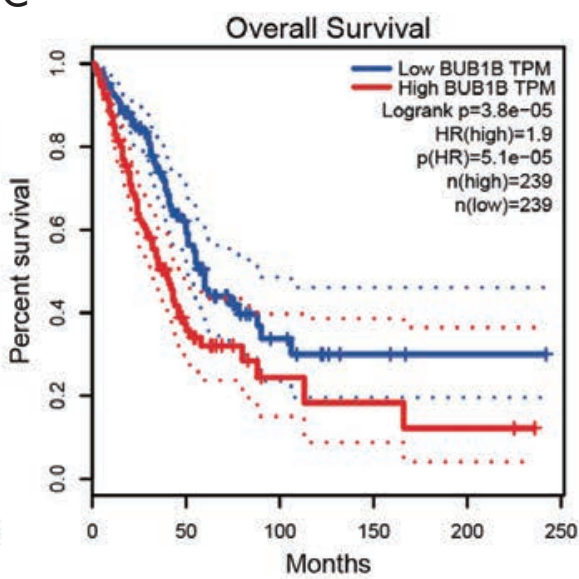

$\mathrm{F}$

Overall Survival

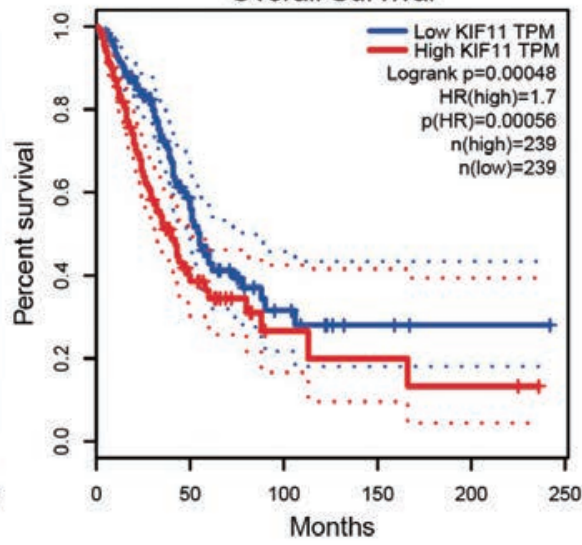

I

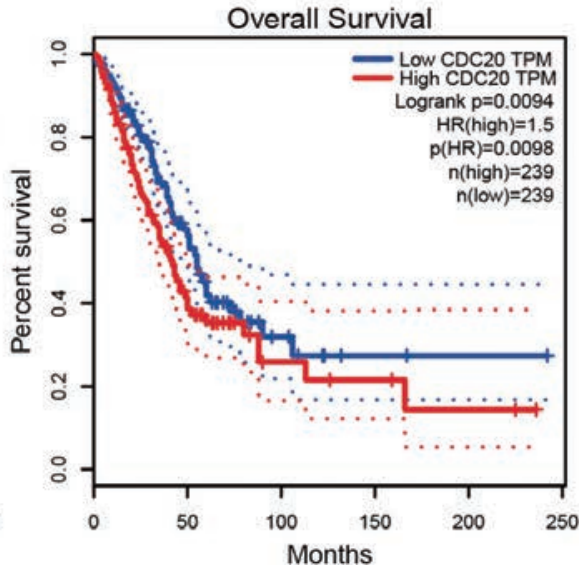

Figure 6 Prognostic value of co-expressed hub genes in lung adenocarcinoma (LUAD) patients. (A) CDK1 (B) BUB1 (C) BUB1B, (D) CCNB1, (E) CCNA2, (F) KIF11, (G) TOP2A, (H) CDC45, (I) CDC20. 
A
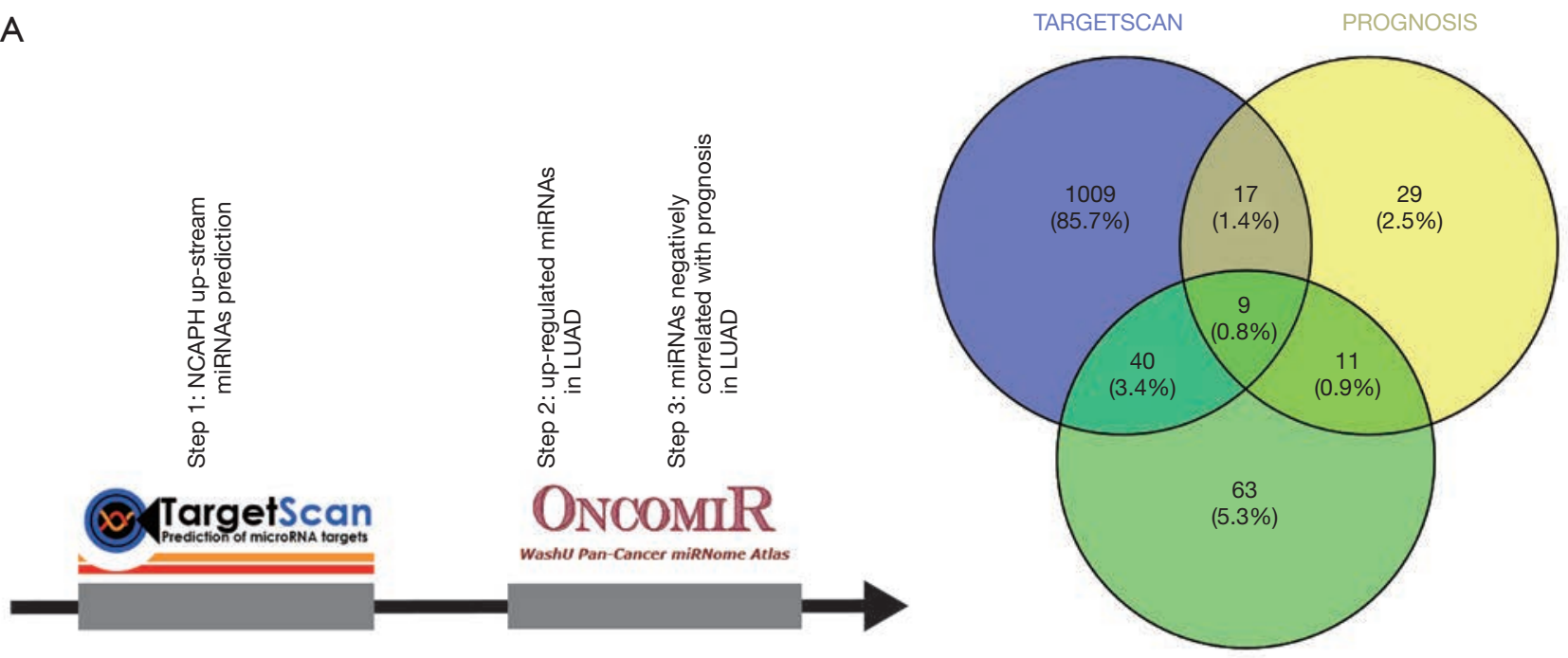

B

C
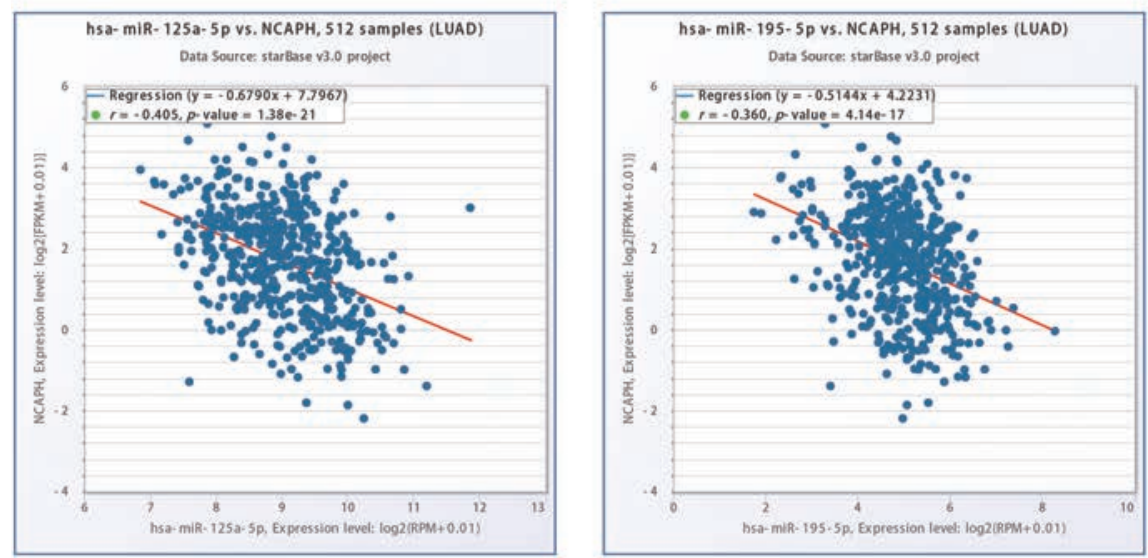

E

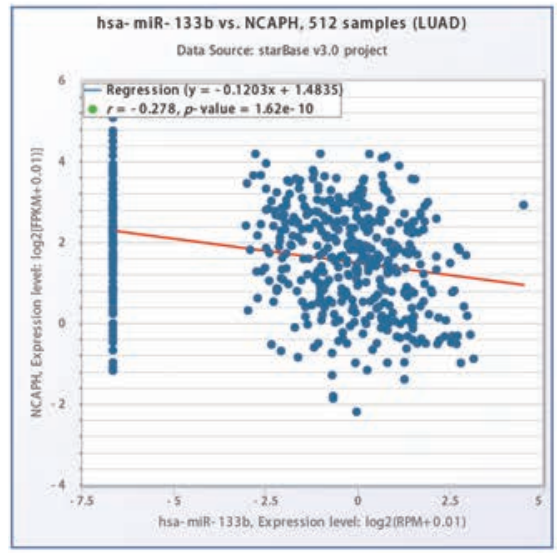

$\mathrm{F}$

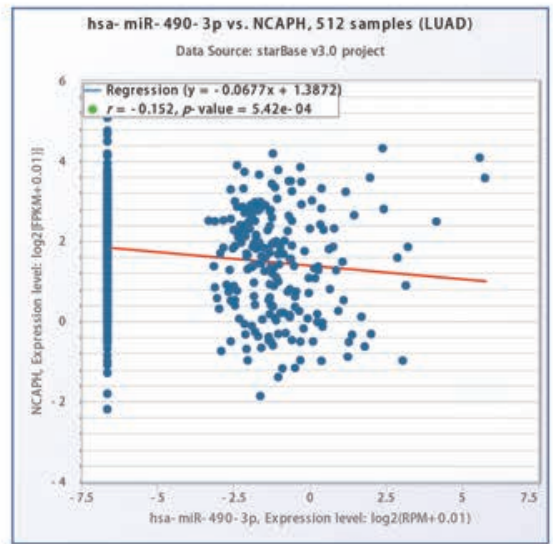

D

EXPRESSION

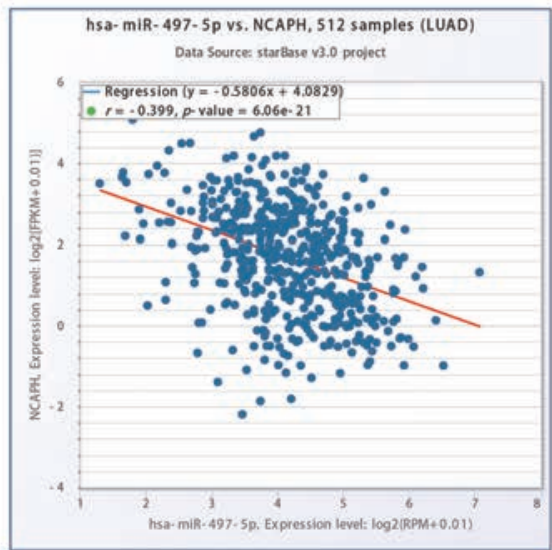

G

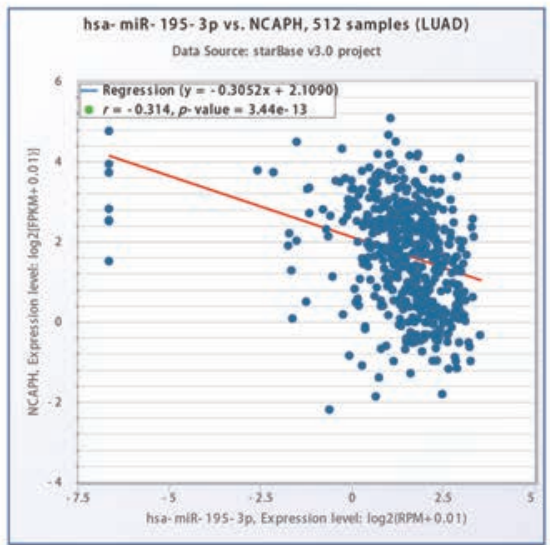


$\mathrm{H}$

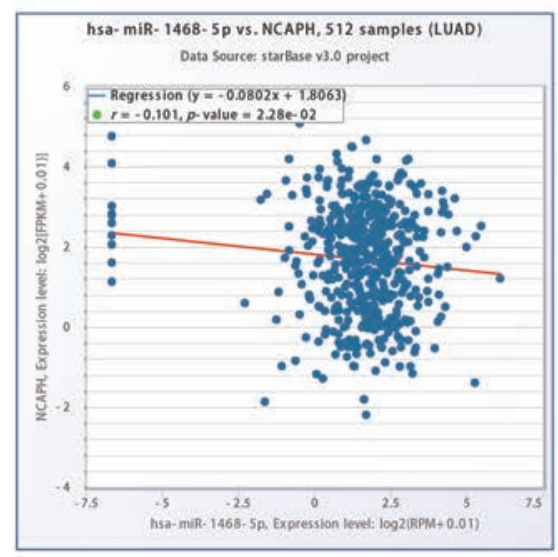

I

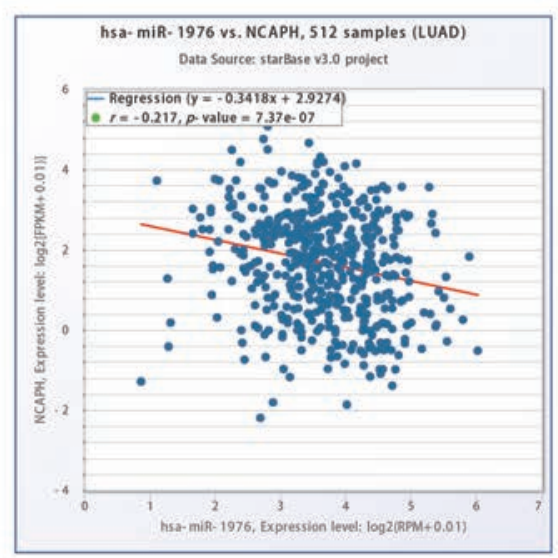

J

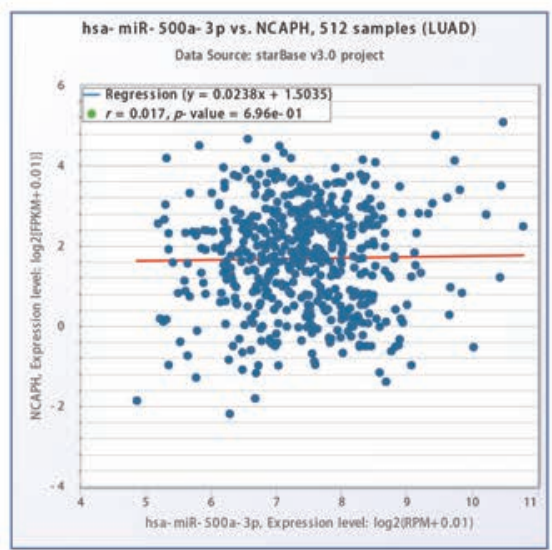

Figure 7 Prediction of miRNAs that regulate NCAPH. (A) The flowchart and Venn picture of the miRNA prediction process. (B,C,D,E,F,G,H,I,J) The expression correlation between NCAHP mRNA and 9 miRNAs in lung adenocarcinoma (LUAD) patients based on ENCORI database. (B) miR-125a-5p, (C) miR-195-5p, (D) miR-497-5p, (E) miR-133b, (F) miR-490-3p, (G) miR-195-3p, (H) miR1468-5p, (I) miR-1976, (J) miR-500a-3p.

Table 2 The prognostic values of 8 potential miRNAs in lung adenocarcinoma (LUAD) analyzed by Oncomir database

\begin{tabular}{|c|c|c|c|c|}
\hline miRNA name & Log rank $P$ value & Deceased Log2 mean expression & Living Log2 mean expression & T-test $P$ value \\
\hline hsa-miR-1468-5p & 1.16E-03 & 1.86 & 2.37 & $3.51 \mathrm{E}-04$ \\
\hline hsa-miR-195-3p & 4.97E-03 & 1.6 & 1.87 & 2.37E-04 \\
\hline hsa-miR-490-3p & 1.23E-02 & 0.1 & 0.6 & $5.47 \mathrm{E}-02$ \\
\hline hsa-miR-497-5p & 1.02E-02 & 4.23 & 4.51 & 7.35E-04 \\
\hline hsa-miR-195-5p & $3.48 \mathrm{E}-02$ & 5.19 & 5.28 & $2.98 \mathrm{E}-01$ \\
\hline hsa-miR-125a-5p & 2.35E-02 & 9.09 & 9.27 & 1.48E-02 \\
\hline
\end{tabular}

New therapeutic target means a lot to the conquer of cancer. Considering the role of NCAPH as a potential therapeutic target, the CTD database was utilized for finding the chemicals which could down-regulate the NCAPH expression level. Five kinds of chemicals were identified based on previous studies in the database. The problem that whether these chemicals could inhibit the LUAD would be answered with our next experiments.

Despite the big data analysis was used in this study, there are still some limitations: Firstly, lack of the validation in clinical LUAD samples. Secondly, the expression levels of proteins are regulated by many factors, such as promoter methylation, transcription factor and ubiquitination $(45,46)$. These factors were not analyzed in our study. Thirdly, the onco-gene role of NCAPH was not testified invitro and in vivo. These shortcomings will be solved in the next experiment.

\section{Conclusions}

In conclusion, we propose that NCAPH expression in LUAD is a poor prognostic indicator. It may play an important role in cell cycle. In the near future, it may be a potential therapeutic target of LUAD. 

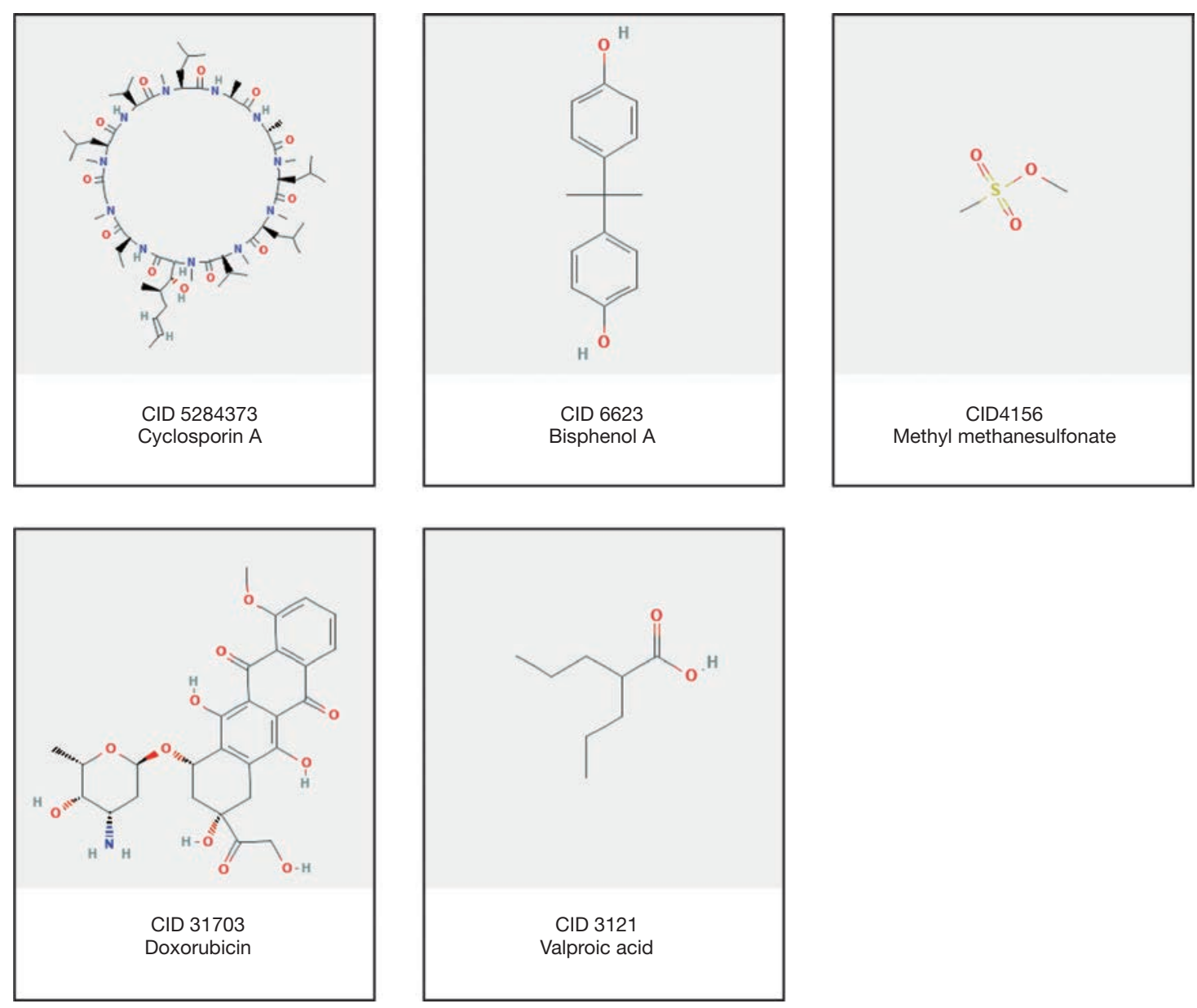

Figure 8 Structure of five chemicals that could decrease the NCAPH.

\section{Acknowledgments}

Funding: We thank financial support from the National Natural Science Foundation of China (No. 81672934).

\section{Footnote}

Reporting Checklist: The authors have completed the TRIPOD reporting checklist. Available at http://dx.doi. org/10.21037/tcr-20-2217

Conflicts of Interest: All authors have completed the ICMJE uniform disclosure form (available at http://dx.doi. org/10.21037/tcr-20-2217). The authors have no conflicts of interest to declare.

Ethical Statement: The authors are accountable for all aspects of the work in ensuring that questions related to the accuracy or integrity of any part of the work are appropriately investigated and resolved. The study was conducted in accordance with the Declaration of Helsinki (as revised in 2013).

Open Access Statement: This is an Open Access article distributed in accordance with the Creative Commons Attribution-NonCommercial-NoDerivs 4.0 International License (CC BY-NC-ND 4.0), which permits the noncommercial replication and distribution of the article with the strict proviso that no changes or edits are made and the original work is properly cited (including links to both the formal publication through the relevant DOI and the license). See: https://creativecommons.org/licenses/by-nc-nd/4.0/.

\section{References}

1. Torre LA, Siegel RL, Jemal A. Lung Cancer Statistics. Adv 
Exp Med Biol 2016;893:1-19.

2. Park CK, Kim SJ. Trends and Updated Statistics of Lung Cancer in Korea. Tuberc Respir Dis (Seoul) 2019;82:175-7.

3. Feng RM, Zong YN, Cao SM, et al. Current cancer situation in China: good or bad news from the 2018 Global Cancer Statistics? Cancer Commun (Lond) 2019;39:22 .

4. Chen W, Zheng R, Baade PD, et al. Cancer statistics in China, 2015. CA Cancer J Clin 2016;66:115-32.

5. Strano S, Lupo A, Lococo F, et al. Prognostic significance of vascular and lymphatic emboli in resected pulmonary adenocarcinoma. Ann Thorac Surg 2013;95:1204-10.

6. Reck M, Rodriguez-Abreu D, Robinson AG, et al. Updated Analysis of KEYNOTE-024: Pembrolizumab Versus Platinum-Based Chemotherapy for Advanced NonSmall-Cell Lung Cancer With PD-L1 Tumor Proportion Score of 50\% or Greater. J Clin Oncol 2019;37:537-46.

7. Ramalingam SS, Vansteenkiste J, Planchard D, et al. Overall Survival with Osimertinib in Untreated, EGFR-Mutated Advanced NSCLC. N Engl J Med 2020;382:41-50.

8. Hirano T. Condensins: universal organizers of chromosomes with diverse functions. Genes Dev 2012;26:1659-78.

9. Schleiffer A, Kaitna S, Maurer-Stroh S, et al. Kleisins: A superfamily of bacterial and eukaryotic SMC protein partners. Mol Cell 2003;11:571-5.

10. Cui F, Hu J, Xu Z, et al. Overexpression of $\mathrm{NCAPH}$ is upregulated and predicts a poor prognosis in prostate cancer. Oncol Lett 2019;17:5768-76.

11. Kim JH, Youn Y, Kim KT, et al. Non-SMC condensin I complex subunit $\mathrm{H}$ mediates mature chromosome condensation and DNA damage in pancreatic cancer cells. Sci Rep 2019;9:17889.

12. Sun C, Huang S, Wang H, et al. Non-SMC condensin I complex subunit $\mathrm{H}$ enhances proliferation, migration, and invasion of hepatocellular carcinoma. Mol Carcinog 2019;58:2266-75.

13. Yin L, Jiang LP, Shen QS, et al. NCAPH plays important roles in human colon cancer. Cell Death Dis 2017;8:e2680.

14. Tang Z, Li C, Kang B, et al. GEPIA: a web server for cancer and normal gene expression profiling and interactive analyses. Nucleic Acids Res 2017;45:W98-W102.

15. Chandrashekar DS, Bashel B, Balasubramanya SAH, et al. UALCAN: A Portal for Facilitating Tumor Subgroup Gene Expression and Survival Analyses. Neoplasia 2017;19:649-58.

16. Hou J, Aerts J, den Hamer B, et al. Gene expressionbased classification of non-small cell lung carcinomas and survival prediction. PLoS One 2010;5:e10312.
17. Su LJ, Chang C, Wu Y, et al. Selection of DDX5 as a novel internal control for Q-RT-PCR from microarray data using a block bootstrap re-sampling scheme. Bmc Genomics 2007;8:140.

18. Landi MT, Dracheva T, Rotunno M, et al. Gene expression signature of cigarette smoking and its role in lung adenocarcinoma development and survival. PLoS One 2008;3:e1651.

19. Pontén F, Schwenk JM, Asplund A, et al. The Human Protein Atlas as a proteomic resource for biomarker discovery. J Intern Med 2011;270:428-46.

20. Nagy Á, Lánczky A, Menyhárt O, et al. Validation of miRNA prognostic power in hepatocellular carcinoma using expression data of independent datasets. Sci Rep 2018;8:9227.

21. Cerami E, Gao J, Dogrusoz U, et al. The cBio cancer genomics portal: an open platform for exploring multidimensional cancer genomics data. Cancer Discov 2012;2:401-4.

22. Huang da W, Sherman BT, Lempicki RA. Systematic and integrative analysis of large gene lists using DAVID bioinformatics resources. Nat Protoc 2009;4:44-57.

23. Szklarczyk D, Gable A, Lyon D, et al. STRING v11: protein-protein association networks with increased coverage, supporting functional discovery in genome-wide experimental datasets. Nucleic Acids Res 2019;47:D607-13.

24. Chin $\mathrm{CH}$, Chen $\mathrm{S}, \mathrm{Wu} \mathrm{H}$, et al. cytoHubba: identifying hub objects and sub-networks from complex interactome. BMC Syst Biol 2014;8 Suppl 4:S11.

25. Agarwal V, Bell G, Nam J, et al. Predicting effective microRNA target sites in mammalian mRNAs. Elife 2015;4:e05005.

26. Wong NW, Chen Y, Chen S, et al. OncomiR: an online resource for exploring pan-cancer microRNA dysregulation. Bioinformatics 2018;34:713-5.

27. Li JH, Liu S, Zhou H, et al. starBase v2.0: decoding miRNA-ceRNA, miRNA-ncRNA and protein-RNA interaction networks from large-scale CLIP-Seq data. Nucleic Acids Res 2014;42:D92-7.

28. Davis AP, Grondin C, Johnson R, et al. The Comparative Toxicogenomics Database: update 2019. Nucleic Acids Res 2019;47:D948-54.

29. Hu J, Zhou L, Song Z, et al. The identification of new biomarkers for bladder cancer: A study based on TCGA and GEO datasets. J Cell Physiol 2019. [Epub ahead of print].

30. Wang Y, Chen L, Wang G, et al. Fifteen hub genes associated with progression and prognosis of clear cell 
renal cell carcinoma identified by coexpression analysis. J Cell Physiol 2019;234:10225-37.

31. Wang J, Wang Y, Kong F, et al. Identification of a six-gene prognostic signature for oral squamous cell carcinoma. J Cell Physiol 2020;235:3056-68.

32. Zhang L, He M, Zhu W, et al. Identification of a panel of mitotic spindle-related genes as a signature predicting survival in lung adenocarcinoma. J Cell Physiol 2020;235:4361-75.

33. Li J, Qian W, Sun Q. Cyclins regulating oocyte meiotic cell cycle progression. Biol Reprod 2019;101:878-81.

34. Zhang S, Tischer T, Barford D. Cyclin A2 degradation during the spindle assembly checkpoint requires multiple binding modes to the APC/C. Nat Commun 2019;10:3863.

35. Tian JH, Mu L, Wang M, et al. BUB1B Promotes Proliferation of Prostate Cancer via Transcriptional Regulation of MELK. Anticancer Agents Med Chem 2020;20:1140-6.

36. Venuto S, Monteonofrio L, Cozzolino F, et al. TRIM8 interacts with KIF11 and KIFC1 and controls bipolar spindle formation and chromosomal stability. Cancer Lett 2020;473:98-106.

37. Guo W, Sun S, Guo L, et al. Elevated TOP2A and $\mathrm{UBE} 2 \mathrm{C}$ expressions correlate with poor prognosis in patients with surgically resected lung adenocarcinoma: a study based on immunohistochemical analysis and bioinformatics. J Cancer Res Clin Oncol 2020;146:821-41.

38. Thomson DW, Dinger M. Endogenous microRNA sponges: evidence and controversy. Nat Rev Genet

Cite this article as: Zhou $\mathrm{W}, \mathrm{Hu} \mathrm{J}$, Zhao J. Non-SMC condensin I complex subunit H (NCAPH), a regulator of cell cycle, predicts poor prognosis in lung adenocarcinoma patients: a study mainly based on TCGA and GEO database. Transl Cancer Res 2020;9(12):7572-7587. doi: 10.21037/tcr-20-2217
2016;17:272-83.

39. Zheng H, Wu J, Shi J, et al. miR-125a-5p upregulation suppresses the proliferation and induces the cell apoptosis of lung adenocarcinoma by targeting NEDD9. Oncol Rep 2017;38:1790-6.

40. Chen G, Hu J, Huang Z, et al. MicroRNA-1976 functions as a tumor suppressor and serves as a prognostic indicator in non-small cell lung cancer by directly targeting PLCE1. Biochem Biophys Res Commun 2016;473:1144-51.

41. Gu H, Yang T, Fu S, et al. MicroRNA-490-3p inhibits proliferation of A549 lung cancer cells by targeting CCND1. Biochem Biophys Res Commun 2014;444:104-8.

42. Li G, Wang K, Wang J, et al. miR-497-5p inhibits tumor cell growth and invasion by targeting SOX5 in non-smallcell lung cancer. J Cell Biochem 2019;120:10587-95.

43. Luo J, Pan J, Jin Y, et al. MiR-195-5p Inhibits Proliferation and Induces Apoptosis of Non-Small Cell Lung Cancer Cells by Targeting CEP55. Onco Targets Ther 2019;12:11465-74.

44. Zhen Y, Liu J, Huang Y, et al. miR-133b Inhibits Cell Growth, Migration, and Invasion by Targeting MMP9 in Non-Small Cell Lung Cancer. Oncol Res 2017;25:1109-16.

45. Li Y, Sun Q, Jiang M, et al. KLF9 suppresses gastric cancer cell invasion and metastasis through transcriptional inhibition of MMP28. FASEB J 2019;33:7915-28.

46. Liu L, Yu L, Zeng C, et al. E3 Ubiquitin Ligase HRD1 Promotes Lung Tumorigenesis by Promoting Sirtuin 2 Ubiquitination and Degradation. Mol Cell Biol 2020;40:e00257-19. 